\title{
Spin constraints on Regge predictions and perturbative evolution in high energy collisions
}

\author{
Steven D. Bass \\ Institute for Theoretical Physics, \\ Universität Innsbruck, Technikerstrasse 25, Innsbruck, A 6020 Austria
}

\begin{abstract}
Two key issues in the application of perturbative QCD and Regge predictions to high energy processes are whether the hard and soft pomerons should be considered as two separate distinct exchanges and whether the Regge intercepts are $Q^{2}$ independent or not. Models involving a distinct hard pomeron exchange predict much larger values for the LHC total cross-section. Here we argue that there is a polarized analogue of this issue in the isovector part of the spin structure function $g_{1}$ and that the spin data appear to favour a distinct hard exchange.
\end{abstract}

PACS: 11.55.-m, 12.40.Nn, 13.60.Hb, 13.88.+e

Keywords: Spin structure functions, Regge phenomenology 
Regge and small $x$ physics has been vigorously studied in the context of HERA and is important for predicting the LHC total cross-section. There are various models and approaches which depend on whether the hard pomeron observed at HERA should be treated as a distinct exchange [1]. Predictions for the LHC total cross-section range from about $90 \mathrm{mb}$ up to about $150 \mathrm{mb}[2,3]$ with the larger values associated with a distinct hard-pomeron [3]. In this paper we address the questions: Can we use present information from polarized processes [4] to help constrain models of Regge and small $x$ dynamics and is there evidence in polarized data for distinct hard exchanges ?

Models of small $x$ physics generally fall into two clases. First consider unpolarized scattering. The first approach [5] involves a soft-pomeron and perturbative QCD evolution (DGLAP, $\alpha_{s}^{n} \ln ^{m} \frac{1}{x} \ldots$ resummation) which drives the increase in the effective intercept $\alpha, F_{2} \sim x^{-\alpha}$, from the soft-pomeron value 0.08 to the value $\sim 0.4$ extracted from HERA data [6]. In a second approach Cudell et al. [7] have argued that the Regge intercepts should be independent of $Q^{2}$ and that the HERA data is described by a distinct hard-pomeron exchange in addition to the soft-pomeron. The hard pomeron should also appear in low $Q^{2}$ photoproduction data and in protonproton collisions. There are two conflicting measurements of the total cross-section at the Tevatron [8]. The larger CDF measurement favours a separate hard pomeron contribution.

Is there a place in polarized data where similar physics issues occur? There are interesting clues in the data. Our knowledge of the $g_{1}$ spin structure function at deep inelastic $Q^{2}$ mostly comes from SLAC small $x$ data between 0.01 and 0.1 for the isovector part of $g_{1}[9]$ and COMPASS small $x$ data between 0.004 and 0.1 for the isoscalar part [10]. The isovector part of $g_{1}$ rises as $\sim x^{-0.5}$ and is much bigger than the isoscalar part of $g_{1}$, which is close to zero in the measured kinematics in sharp contrast to the unpolarized structure function $[11,4,12]$. In this paper we focus on the isovector part of $g_{1}$.

The rise in $g_{1}^{p-n}=g_{1}^{p}-g_{1}^{n}$ is a challenge for Regge predictions and perturbative QCD. The Regge prediction for $g_{1}^{p-n}$ at small $x$ is

$$
g_{1}^{p-n} \sim \sum_{i} f_{i}\left(\frac{1}{x}\right)^{\alpha_{i}} .
$$

Here the $\alpha_{i}$ denote the Regge intercepts for isovector $a_{1}$ Regge exchange and the $a_{1}$-pomeron cuts [13]. The coefficients $f_{i}$ are to be determined from experiment. If one makes the usual assumption that the $a_{1}$ Regge trajectories are straight lines parallel to the $(\rho, \omega)$ trajectories then one finds $\alpha_{a_{1}} \simeq-0.4$ for the leading trajectory, within the phenomenological range $-0.5 \leq \alpha_{a_{1}} \leq 0$ discussed in Ref. 14. Taking the masses of the $a_{1}(1260)$ and $a_{3}(2070)$ states together with the $a_{1}(1640)$ and $a_{3}(2310)$ states from the Particle Data Group [15] yields two parallel $a_{1}$ trajectories with slope 
$\sim 0.75 \mathrm{GeV}^{-2}$ and a leading trajectory with slightly lower intercept: $\alpha_{a_{1}} \simeq-0.18$. For this value of $\alpha_{a_{1}}$ the effective intercepts corresponding to the $a_{1}$ soft-pomeron cut and the $a_{1}$ hard-pomeron cut are $\simeq-0.1$ and $\simeq+0.22$ respectively if one takes the soft and hard pomerons as two distinct exchanges ${ }^{1}$ Values of $\alpha_{a_{1}}$ close to zero could be achieved with curved Regge trajectories; the recent model of Brisudova et al. [16] predicts $\alpha_{a_{1}}=-0.03 \pm 0.07$. For this value the intercepts of the $a_{1}$ softpomeron cut and the $a_{1}$ hard-pomeron cut become $\sim+0.05$ and $\sim+0.37$. The $a_{1}$ and $a_{1}$ soft-pomeron cut alone are unable to account for the data. ${ }^{2}$

The observed rise in $g_{1}^{p-n}$ at deep inelastic values of $Q^{2}$ is required to reproduce the area under the fundamental Bjorken sum rule [17]

$$
\begin{array}{rl}
\int_{0}^{1} & d x g_{1}^{(p-n)}\left(x, Q^{2}\right) \\
& =\frac{g_{A}^{(3)}}{6}\left[1-\frac{\alpha_{s}\left(Q^{2}\right)}{\pi}-3.583\left(\frac{\alpha_{s}\left(Q^{2}\right)}{\pi}\right)^{2}-20.215\left(\frac{\alpha_{s}\left(Q^{2}\right)}{\pi}\right)^{3}+\ldots\right]
\end{array}
$$

Here $g_{A}^{(3)}=1.2695 \pm 0.0029$ [15] is the scale-invariant isovector axial-charge measured in neutron beta-decays. The sum-rule has been confirmed in polarized deep inelastic scattering experiments at the level of $10 \%$ [18]. About $50 \%$ of the Bjorken sum-rule comes from $x$ values less than about 0.12 and $10 \%$ comes from $x$ values less than $0.01[4,11,9]$. The $g_{1}^{p-n}$ data is consistent with quark model and perturbative QCD predictions in the valence region $x>0.2$ [19]. The size of $g_{A}^{(3)}$ forces us to accept a large contribution from small $x$ and the observed rise in $g_{1}^{p-n}$ is required to fulfil this non-perturbative constraint.

Does this rise follow from $a_{1}$ exchange plus perturbative QCD evolution or is there a distinct hard exchange ? - that is, a polarized analogue of the one or two pomerons question! The difference between the effective intercept describing $g_{1}^{p-n}$ at deep inelastic values of $Q^{2}$ and the prediction based on soft $a_{1}$ exchange is a factor of up to 2-3 bigger than the difference in the effective intercept needed to describe $F_{2}$ in the unpolarized HERA data and the soft-pomeron prediction.

In the conventional approach the $a_{1}$ term (or $a_{1}$ soft-pomeron cut) should describe the high-energy part of $g_{1}$ close to photoproduction and provide the input for perturbative QCD evolution at deep inelastic values of $Q^{2}$ above the transition region. One then applies perturbative QCD (DGLAP or DGLAP plus double logarithm $\ln ^{2} \frac{1}{x} \ldots$ resummation) and out should come the rising structure function seen in the data $[20,21,22,23]$. For $g_{1}^{p-n}$ with DGLAP evolution this approach has the

\footnotetext{
${ }^{1}$ I thank P.V. Landshoff for valuable discussions on this issue.

${ }^{2}$ It should be noted that, in the measured $x$ range, the effective isovector exponent 0.5 could be softened through multiplication by a $(1-x)^{n}$ factor - for example associated with perturbative QCD counting rules at large $x$ ( $x$ close to one). For example, the exponent $x^{-0.5}$ could be modified to about $x^{-0.25}$ through multiplication by a factor $(1-x)^{6}$. However, this is not sufficient to reconcile the measured rising structure function with the naive Regge prediction involving soft $a_{1}$ exchange.
} 
challenging feature that the input and output (at soft and hard scales) are governed by non-perturbative constraints with perturbative QCD evolution in the middle unless the $a_{1}$ Regge input has information about $g_{A}^{(3)}$ built into it. (Furthermore, perturbative $\alpha_{s}^{l+1} \ln ^{2 l} x$ resummation calculations predict a sharp rise [21, 22, 23] $\sim x^{-\gamma}$ with $\gamma \sim 0.9-1$ in the absolute value of the isosinglet spin structure function $g_{1}^{d}$ which is not observed in the present data. The measured structure function is consistent with zero for $x$ between the lowest value 0.004 and 0.05 [10], and the integral $\int_{x_{\min }}^{1} d x g_{1}^{d}$ is observed to converge within the errors for $x_{\min } \sim 0.05$ [24].) The alternative scenario is a separate hard-exchange contribution (perhaps an $a_{1}$ hard-pomeron cut) in addition to the soft $a_{1}$.

Some guidance may come from looking at the QCD evolution equations in moment space. We first consider the perturbative DGLAP approach since this is presently the prime tool used to analyse polarized deep inelastic data. To test deep inelastic sum-rules it is necessary to have all data points at the same value of $Q^{2}$. In the experiments the different data points are measured at different values of $Q^{2}$, viz. $x_{\text {expt. }}\left(Q^{2}\right)$. Next-to-leading order (NLO) QCD-motivated fits taking into account the scaling violations associated with perturbative QCD are used to evolve all the data points to the same $Q^{2}$ using DGLAP evolution [10, 25]. The results of these fits are then extrapolated to $x \sim 0$ to test spin sum-rules.

Let $\Delta q_{3}(x, t)=(\Delta u-\Delta d)(x, t)$ denote the isovector spin-dependent parton distribution with $t=\ln \frac{Q^{2}}{\Lambda^{2}} ; \int_{0}^{1} d x \Delta q_{3}(x, t)=g_{A}^{(3)}$. The DGLAP equation for $\Delta q_{3}(x, t)$ is

$$
\frac{d}{d t} \Delta q_{3}(x, t)=\int_{x}^{1} \frac{d y}{y} P\left(\frac{x}{y}\right) \Delta q_{3}(y, t)
$$

where

$$
P(z)=\frac{\alpha_{s}(t)}{2 \pi} C_{2}(R)\left[\frac{1+z^{2}}{(1-z)_{+}}+\frac{3}{2} \delta(z-1)\right]
$$

is the leading-order spin-dependent splitting function; $C_{2}(R)=\frac{4}{3}$ and $P(z)$ goes to a constant as $z \rightarrow 0$. The area under $\Delta q_{3}(x, t)$ is conserved because of the Bjorken sum-rule. DGLAP evolution [26] acts to shift the weight of the distribution and $g_{1}^{p-n}$ to smaller $x$ with increasing $Q^{2}$, meaning that a convergent input will be unstable to DGLAP evolution at a given value of small $x$ [27] and prompting the question at what values of $Q^{2}$ and small $x$ should spin-dependent Regge predictions work in this approach, if any?

The evolution equation (3) becomes "singular" in the $x \rightarrow 0$ limit if $\Delta q_{3}$ behaves as a constant for $x \rightarrow 0$ :

$$
\text { convolution } \sim \int_{x}^{1} \frac{d y}{y}\{\ldots\} .
$$

This compares with the singlet channel in unpolarized scattering where the splitting matrix has a $1 / z$ singularity as $z$ goes to zero for evolution into gluons. If the 
unpolarized gluon distribution were to have a leading $1 / y$ pole then the contribution

$$
P\left(\frac{x}{y}\right) g(y) \sim \frac{y}{x} \cdot \frac{1}{y}
$$

would yield the same structure in the evolution equation.

Take the Mellin transform $\int_{0}^{1} d z z^{N-1} P(z)$ :

$$
P\left(N, \alpha_{s}\left(Q^{2}\right)\right)=\int_{0}^{1} d z z^{N-1} P(z)=\frac{\alpha_{s}}{2 \pi} C_{2}(R)\left[-\frac{1}{2}+\frac{1}{N(N+1)}-2 \sum_{j=2}^{N} \frac{1}{j}\right] .
$$

The zeroth moment of the DGLAP splitting function has a pole at $N=0$ at leading order (LO) plus higher-order poles at NLO [28]. If we require that the scattering amplitude is analytic in $Q^{2}$ [29], then Regge singularities are independent of $Q^{2}$ and new singularities should not suddenly appear as $Q^{2}$ increases. This result has practical consequences for singularities in the Mellin transform of the DGLAP splitting function: the $N=0$ poles become an artifact of the perturbative expansion. That is, they should vanish in a full (non-perturbative) resummation [7] otherwise one will generate an unphysical fixed pole $\alpha=0$ contribution in the isovector part of $g_{1}$ as soon as one reaches large enough $Q^{2}$ to apply DGLAP evolution. To see this, consider the Mellin transform of the spin dependent parton distribution

$$
u\left(N, Q^{2}\right)=\int_{0}^{1} d x x^{N-1} \Delta q_{3}\left(x, Q^{2} / \Lambda^{2}\right)
$$

and its DGLAP equation

$$
\frac{\partial}{\partial t} u\left(N, Q^{2}\right)=P\left(N, \alpha_{s}\left(Q^{2}\right)\right) u\left(N, Q^{2}\right) .
$$

If the twist-two term $u\left(N, Q^{2}\right)$ has no pole at $N=0$ at $Q^{2}$ values close to photoproduction, then the solution to the DGLAP equation

$$
u\left(N, Q^{2}\right)=\exp \left[C \log \frac{\log \left(Q^{2} / \Lambda^{2}\right)}{\log \left(Q_{0}^{2} / \Lambda^{2}\right)} P(N)\right] u\left(N, Q_{0}^{2}\right)
$$

automatically generates an essential singularity in $u\left(N, Q^{2}\right)$ at $N=0$ as soon as $Q^{2}$ becomes large enough for the application of perturbative QCD; $C=6 /(33-2 f)$ where $f$ is the number of active flavours. This is not allowed if we assume that $u\left(N, Q^{2}\right)$ is analytic at $N=0$ for some finite range of $Q^{2}$ [7, 29]. It cannot suddenly acquire a fixed singularity at $N=0$ when it is analytically continued in $Q^{2}$. To help understand $P(N)$, Donnachie and Landshoff [30] consider the example of the analogous expansion of the function $\psi\left(N, \alpha_{s}\right)=\sqrt{N^{2}+\alpha_{s}}-N=\frac{\alpha_{s}}{2 N}-\frac{\alpha_{s}^{2}}{8 N^{3}}+\ldots$. Although each term in the expansion is singular at $N=0$, the function $\psi$ is not: the expansion is valid only for $|N|>\alpha_{s}$. (Related issues in unpolarized scattering are discussed in Ref. 32 where a new small $x$ splitting function has been proposed with no $1 / N$ pole in the Mellin transform.) 
The first moment of $P(z)$ vanishes, $P\left(1, \alpha_{s}\right)=0$, corresponding to the conserved axial-charge $g_{A}^{(3)}$. The positive odd moments of the DGLAP splitting function correspond to the anomalous dimensions of axial-tensor operators in the light-cone operator product expansion for deep inelastic scattering. There are no operators corresponding to the poles at $N=0$ or $N=-1$.

Going beyond DGLAP evolution, Blümlein and Vogt [20] have considered the resummation of $\alpha_{s}^{l+1} \ln ^{2 l} x$ terms in the evolution kernels of non-singlet contributions to $g_{1}$. An all orders resummation of these terms in perturbation theory leads only to corrections of $1 \%$ for $g_{1}^{p-n}$ relative to NLO calculations in the kinematics accessible to present experiments. The most singular contributions in the perturbation expansion behave like a power series in $N\left(\alpha_{s} / N^{2}\right)^{k}$ when we take the Mellin transform and work with the moments. One assumes that $\alpha_{s}<3 \pi N^{2} / 8-$ see Eq.(13) of Ref. 21 with the number of colours $N_{c}=3$. Like for the DGLAP procedure discussed above, each term in the perturbative expansion is singular for $N=0$ in moment space. One again encounters the issue of whether the isovector $g_{1}^{p-n}$ structure function is analytic at $N=0$ for finite $Q^{2}$.

Motivated by this discussion we consider a hard exchange "input" to perturbative evolution. For a fixed power behaviour $u(x, t) \sim f\left(Q^{2}\right) x^{-\epsilon}$ the Mellin transform is $u\left(N, Q^{2}\right) \sim \frac{f\left(Q^{2}\right)}{N-\epsilon}$. Substituting this into the DGLAP equation and equating the coefficient of the pole gives an equation for the coefficent of the Regge exponent:

$$
\frac{\partial}{\partial t} f\left(Q^{2}\right)=P\left(N=\epsilon, \alpha_{s}\left(Q^{2}\right)\right) f\left(Q^{2}\right)
$$

If there is a hard exchange with fixed intercept away from the pole at $\epsilon=0$, e.g. $\epsilon=0.5$ or perhaps $\sim 0.2$ for the $a_{1}$ hard-pomeron cut (plus $(1-x)^{n}$ counting rules factors still at work in the measured $x$ range), then a combined Regge-DGLAP approach should be a good approximation - just as a distinct hard pomeron would resolve challenging issues in the interpretation of the unpolarized structure function. (A rising "input" $g_{1}^{p-n} \sim x^{-0.2}$ was used in Ref. 21 at the input scale $Q_{0}^{2}=4 \mathrm{GeV}^{2}$, safely away from the pole at $\epsilon=0$.) Further, if the intercept is $Q^{2}$ independent the issue of reconciling the Regge input to perturbative QCD evolution and the Bjorken sum rule constraint would be resolved. The hard exchange could be looked for in low $Q^{2}$ data - see below.

In the isosinglet sector it is harder to draw firm conclusions. $g_{1}^{p+n}$ is small and consistent with zero in the measured small $x$ kinematics [10]. The Regge prediction involves a contribution $\sim\left\{2 \ln \frac{1}{x}-1\right\}$ from two non-perturbative gluon exchange $[32,33]$ plus contributions from the $f_{1}$ trajectory and $f_{1}$-pomeron cuts. It is unknown whether the gluon exchange contribution Reggeizes or whether it is a fixed pole. Brodsky et al. [34] have argued that colour coherence forces $\Delta g(x) / g(x) \propto x$ when $x \rightarrow 0$. In this scenario we might also expect a polarized version of the hard pomeron with intercept $\sim+0.4$ which would correspond to a rising term (in absolute value) 
like $x^{-0.4}$ as $x \rightarrow 0$. Perhaps the coefficients of these terms are separately suppressed or perhaps they cancel in the measured kinematics?

To summarize, if we assume analyticity in $Q^{2}$ then the isovector spin structure

function $g_{1}^{p-n}$ favours a hard exchange contribution at small $x$ with a $Q^{2}$ independent Regge intercept. This exchange should also contribute to and could be looked for in high-energy polarized photoproduction and in the transition region between $Q^{2}=0$ and deep inelastic values of $Q^{2}\left(Q^{2}<1 \mathrm{GeV}^{2}\right)$. High-energy polarized photoproduction and the transition region could be investigated using a polarized electron-proton collider [35] or perhaps through measurement of low $Q^{2}$ asymmetries at COMPASS using a proton target. A hard exchange contribution might also show up in the spindependent part of the proton-proton total cross-section. In polarized proton-proton collisions one would be looking for a leading behaviour $\Delta \sigma \sim s^{-0.5}$ to $\sim s^{-0.8}$ instead of the simple $a_{1}$ prediction $\sim s^{-1.4}$ and non-perturbative gluon-exchange contribution $\sim\left(\ln s / \mu^{2}\right) / s$ with $\mu \sim 0.5-1 \mathrm{GeV}$ a typical hadronic scale [32, 33]. Will these processes exhibit evidence of a hard exchange with Regge intercept $\alpha \sim+0.5$ or just the exchanges predicted by soft Regge theory? These spin measurements, together with the total cross-section at the LHC, would help constrain our understanding of hard and soft exchanges in high energy collisions.

\section{Acknowledgments}

I thank W. Guryn and P.V. Landshoff for helpful conversations. This research is supported by the Austrian Science Fund (grant P17778-N08).

\section{References}

[1] A. Donnachie and P.V. Landshoff, Phys. Lett. B437 (1998) 408.

[2] R.M. Godbole, A. Grau, R. Hegde, G. Pancheri and Y. Srivastava, Pramana 66 (2006) 657.

[3] P.V. Landshoff, hep-ph/0509240.

[4] S.D. Bass, Rev. Mod. Phys. 77 (2005) 1257.

[5] A.D. Martin, R.G. Roberts, W.J. Stirling and R.S. Thorne, Eur. Phys. J C18 (2000) 117; CTEQ Collaboration: J. Pumplin et al., JHEP 0207 (2002) 012.

[6] H1 Collaboration: C. Adloff et al., Nucl. Phys. B497 (1997) 3; ZEUS Collaboration: J. Breitweg et al., Phys. Lett. B407 (1997) 432.

[7] J.R. Cudell, A.Donnachie and P.V. Landshoff, Phys. Lett. B448 (1999) 281; A. Donnachie and P.V. Landshoff, Phys. Lett. B533 (2002) 277. 
[8] E710 Collaboration: N. Amos et al., Phys. Lett. B243 (1990) 158; CDF Collaboration: F. Abe et al., Phys. Rev. D50 (1994) 5550.

[9] E154 Collaboration: K. Abe et al., Phys. Rev. Lett. 79 (1997) 26.

[10] COMPASS Collaboration: V.Yu. Alexakhin et al., hep-ex/0609038

[11] J. Soffer and O.V. Teryaev, Phys. Rev. D56 (1997) 1549.

[12] S.D. Bass and M.M. Brisudova, Eur. Phys. J. A4 (1999) 251.

[13] R.L. Heimann, Nucl. Phys. B64 (1973) 429.

[14] J. Ellis and M. Karliner, Phys. Lett. B213, 73 (1988).

[15] The Particle Data Group: S. Eidelmann et al., Phys. Lett. B592 (2004) 1.

[16] M.M Brisudova, L. Burakovsky and T. Goldman, Phys. Rev. D61 (2000) 054013.

[17] J.D. Bjorken, Phys. Rev. 148 (1966) 1467; Phys. Rev. D1 (1970) 1376.

[18] SMC Collaboration: B. Adeva et al., Phys. Rev. D58 (1998) 112002;

E155 Collaboration: P.L. Anthony et al., Phys. Lett. B463 (1999) 339.

[19] S.D. Bass, Eur. Phys. J. A5 (1999) 17.

[20] J. Blümlein and A. Vogt, Phys. Lett. B370 (1996) 149.

[21] B. Badelek and J. Kwiecinski, Phys. Lett. B418 (1998) 229; J. Kwiecinski and B. Ziaja, Phys. Rev. D60 (1999) 054004; B. Ziaja, Acta Phys. Pol. B 34 (2003) 3013.

[22] J. Bartels, B.I. Ermolaev and M.G. Ryskin, Z. Phys. C70 (1996) 273; Z. Phys. C72 (1996) 627.

[23] B.I. Ermolaev, M. Greco and S.I. Troyan, Nucl. Phys. B594 (2001) 71; Phys. Lett. B579 (2004) 321; Phys. Lett. B622 (2005) 93.

[24] HERMES Collaboration: A. Airapetian et al., hep-ex/0609039.

[25] SMC Collaboration: B. Adeva et al., Phys. Rev. D58 (1998) 112002; E-155 Collaboration: P.L. Anthony et al., Phys. Lett. B493 (2000) 19; T. Gehrmann and W.J. Stirling, Phys. Rev. D53 (1996) 6100; G. Altarelli, R.D. Ball, S. Forte and G. Ridolfi, Nucl. Phys. B496 (1997) 337; M. Glück, E. Reya, M. Stratmann and W. Vogelsang, Phys. Rev. D63 (2001) 094005; J. Blümlein and H. Böttcher, Nucl. Phys. B636 (2002) 225; Y. Goto et al., Phys. Rev. D62 (2000) 034017; M. 
Hirai et al., Phys. Rev. D69 (2004) 054021; E. Leader, A.V. Sidorov and D.B. Stamenov, Eur. Phys. J. C23 (2002) 479.

[26] G. Altarelli and G. Parisi, Nucl. Phys. B126 (1977) 298.

[27] G. Altarelli, R. Ball, S. Forte and G. Ridolfi, Nucl. Phys. B496 (1997) 337.

[28] M. Glück, E. Reya, M. Stratmann and W. Vogelsang, Phys. Rev. D53 (1996) 4775 .

[29] R.J. Eden, P.V. Landshoff, D.I. Olive and J.C. Polkinghorne, The Analytic S-Matrix, Cambridge University Press, 2002.

[30] A. Donnachie and P.V. Landshoff, Acta Phys. Pol. B34 (2003) 2989.

[31] G. Altarelli, R.D. Ball and S. Forte, Nucl. Phys. B742 (2006) 1.

[32] S.D. Bass and P.V. Landshoff, Phys. Lett. B336 (1994) 537.

[33] F.E. Close and R.G. Roberts, Phys. Lett. B336 (1994) 257.

[34] S.J. Brodsky, M. Burkardt and I. Schmidt, Nucl. Phys. B441 (1995) 197.

[35] S.D. Bass and A. De Roeck, Eur. Phys. J. C18 (2001) 531. 